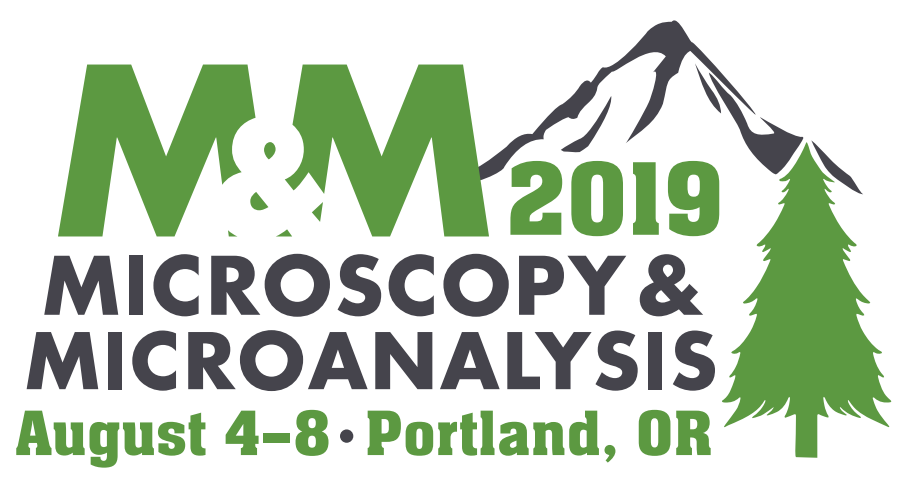

\title{
Microscopy \& Microanalysis 2019 in Portland, Oregon
}

\author{
Alice Dohnalkova, Program Chair \\ Pacific Northwest National Laboratory, Environmental Molecular Sciences Laboratory, Richland, WA 99352 \\ alice.dohnalkova@pnnl.gov
}

The Microscopy Society of America (MSA), the Microanalysis Society (MAS), and the International Field Emission Society (IFES) cordially invite you to attend and present your research at Microscopy \& Microanalysis 2019 in Portland, Oregon. As many of you who have previously visited Portland know, this city is a terrific location for our meeting: set between Mt. Hood and the Pacific Coast, Portland (PDX for short) presents a variety of attractions for everybody. Infused with the awesome Pacific Northwest mentality of environmental conservation and organic local food sourcing, you will find a lot of wonderful restaurants here, including vegetarian, vegan, and gluten-free options, plus a myriad of microbrews. Don't forget to visit famous PDX staples like Powell's Books and the charismatic Voodoo donut shop. Portland offers tax-free shopping. If you have an extra half-day, take a winery tour in the Willamette Valley countryside or a drive to the Pacific Coast's Cannon beach. Make sure to bring the right footwear if you're planning to explore the great outdoors like the Columbia Gorge area with its numerous waterfalls.

Besides visiting the wonderful Portland area, the main attraction will be the M\&M-and it will be memorable this year! Once again, the latest and most innovative applications and instrumentation developments will be presented by from hundreds of investigators using microscopy and microanalysis techniques in the biological and physical sciences. The M\&M 2019 meeting will feature close to 40 symposia covering a broad range of topics, numerous educational opportunities, and the microscopy outreach program, ProjectMICRO. Prior to the meeting, there will be Sunday pre-meeting short courses and two pre-meeting congresses. The annual microscopy exhibition again will be the largest in the world, with 120 companies from the USA and 12 other countries. Social activities will include the opening reception and daily refreshments served during our popular poster sessions. Prize-winning posters will be announced each day at the end of the session.

We are excited and honored to feature two Nobel Laureates as our plenary lecturers. Our first plenary speaker will be
Dr. Joachim Frank, Professor of Biochemistry and Molecular Biophysics and of Biological Sciences at Columbia University, New York. He shared with Jacques Dubochet and Richard Henderson the 2017 Nobel Prize in Chemistry for "Developing cryo-electron microscopy for the highresolution structure determination of biomolecules in solution." Dr. Frank's major contribution to the field has been in developing mathematical and computational methods for processing and analyzing cryo-EM images of multiple randomly oriented molecules within a sample and compiling them into a representative 3D structure. Dr. Frank used his algorithms to generate the first 3D images of the ribosome, a large

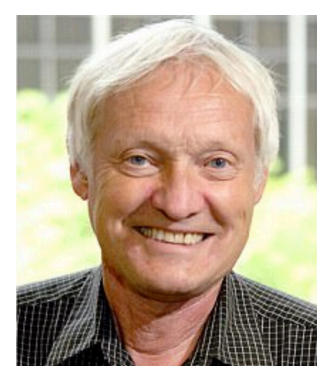

Dr. Joachim Frank structure made of several proteins and RNA strands, which is responsible for translating RNA into proteins inside cells in all organisms. These algorithms, when combined with Dubochet's method of ice-embedding, produced information on conformational changes of macromolecules in their native states, enabling a deeper understanding of the way "molecular machines" function in cells. Structures can now be determined of many molecules that resist crystallization and hence cannot be studied by X-ray crystallography. Initially, the resolution that could be obtained was limited by the poor performance of recording media. This technical problem was solved seven years ago with the introduction of cameras capable of detecting single electrons. The development of cryo-electron microscopy has revolutionized the imaging of biomolecules and propelled biochemistry into a new era. By now, about 1,500 structures of proteins and RNA-protein complexes have been solved and entered in a public database, making an increasingly important contribution to molecular medicine and the development of drug therapies. Dr. Frank's achievements were earlier recognized with MSA's Distinguished Biological Scientist Award in 2003. 
Our second plenary speaker will be Dr. Richard Henderson of the Medical Research Council Laboratory of Molecular Biology (MRC LMB), Cambridge, UK. Dr. Henderson was awarded the Nobel Prize in Chemistry 2017, together with Jacques Dubochet and Joachim Frank, for "Developing cryo-electron microscopy for the high-resolution structure determination of biomolecules in solution." He will present a talk titled "Single particle cryoEM: potential for further improvement." Dr. Henderson developed TEM into a tool for the direct determination of the structure of proteins, and he applied it most notably to two-dimensional (2D) crystals of the purple light-harvesting protein, bacteriorhodopsin. Images and electron diffraction patterns of many 2D crystals of bacteriorhodopsin from multiple angles were acquired using low-dose electron exposures, and combined to generate a 3D image of the protein. $\mathrm{He}$ continued to refine this technique over many years until he produced images

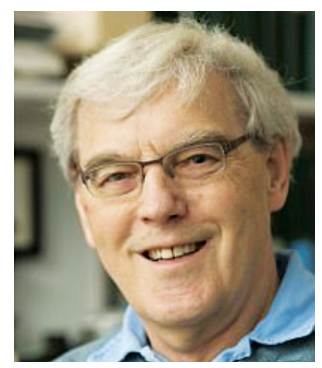

Dr. Richard Henderson at similar resolutions as those obtained from X-ray diffraction. Later, Dr. Henderson turned his attention to the development and improvement of methods of high-resolution electron cryo-microscopy and single particle structure determination. With colleagues, he advanced these techniques for exploring high-resolution ultrastructure of membrane proteins, protein complexes, and other non-crystalline biomolecules in solution. During this journey, Dr. Henderson made critical contributions to many single-particle electron microscopy approaches, including pioneering the development of direct electron detectors. Dr. Henderson was presented with MSA's Distinguished Biological Scientist Award in 2005.

As usual, M\&M 2019 will present a great variety of symposia topics in analytical, life, and physical sciences. This year, we have a special symposium: "Cryo-EM - from Physics to Cell Biology: Honoring the Remarkable Legacy of Ken Downing." It will showcase the late Dr. Downing's work in single-particle cryo-EM, cryo-electron tomography, and the microscopy methods he used for his seminal discoveries. Dr. Downing, MSA Fellow and former MSA president, was enormously influential in the field of electron microscopy. His scientific accomplishments span an amazing range of widely recognized techniques and methods. Work in his laboratory led to the first high-resolution structure of tubulin using electron crystallography, and he made important contributions to the current "resolution revolution" in single-particle cryo-EM.

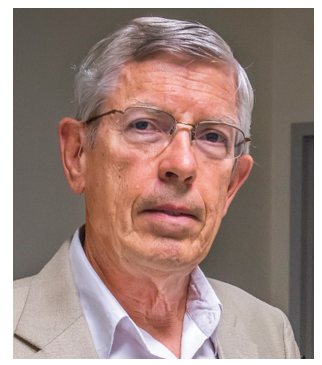
Dr. Kenneth Downing (1945-2018)
Perhaps even more importantly, Ken Downing was a wonderful mentor to the next generation of scientists.

Preceding the M\&M 2019 technical program, we will have two pre-meeting congresses. First, "NexTEM: Next-generation Transmission Electron Microscopy - Beyond Current Limits of Resolution, Environments, and Data Analysis" will include presentations and a discussion forum about integration of advanced and emerging instrumentation, as well as approaches to examine nanoscale systems with high spatial resolution and chemical sensitivity, novel in situ and operando methods to study the dynamics of complex materials systems, and data analysis tools and machine learning approaches. Secondly, the "Third Annual Pre-Meeting Congress for Students, Post-Docs, and Early-Career Professionals in Microscopy and Microanalysis," organized by the MSA Student Council, will provide a forum to share research and data in an engaging, non-intimidating, and interactive setting. This event provides professional networking among fellow young microscopists and career development mentoring from recent graduates. The Friday evening social, Saturday lunch, and evening banquet are included with registration.

Education and training are important components of the M\&M technical program each year, and 2019 is no exception. Six Sunday short courses on material science, biology, and data analysis will be presented. Additional opportunities are available in tutorials in biology and physical sciences during the meeting week. This year, the tutorials will cover the cutting-edge subjects of machine learning, electron ptychography, electron optics for cryoEM, and the computational toolbox for cryoEM, to name just a few. The Technologists' Forum will present invited experts in two roundtable discussions. In "Technical Careers in Microscopy - For the Love of Microscopy," a panel of technologists, including early- and late-career technologists from academia and industry, will discuss their careers in microscopy and how they evolved through the years. Learn how to become a Certified Electron Microscopy Technologist (CEMT) and what it can do for your career. Another roundtable discussion will focus on "Utilization of the National NIH funded Cryo-EM Centers: Transformative High Resolution Cryo-Electron Microscopy," with scientists from three national cryo-EM centers discussing various aspects of cryo-EM techniques.

Finally, our popular microscopy outreach workshop "ProjectMICRO" will be in the MegaBooth all week. See different microscope systems and learning stations for use in the classroom, peruse the books suitable for elementary school age children, and share your experiences of how you have fun with microscopy outreach.

For a complete description of the technical program and educational opportunities, please go to http://www.microscopy.org/MandM/2019. The Call for Papers and other aspects of the program and the meeting are provided under the "Scientific Program" button.

The Executive Program Committee welcomes you to Portland for an excellent and compelling Microscopy \& Microanalysis 2019. Catch up with old friends and make new ones! Look for opportunities to attend society business meetings and socials during the week. On behalf of MSA, MAS, IFES, in cooperation with over a hundred symposia organizers and countless volunteers, we look forward to seeing you in Portland this August! 